\title{
Francisation et évangelisation des Amérindiens de la Nouvelle-France au XVIIe siècle
}

\section{Cornelius J. Jaenen}

Volume 35, 1968

URI : https://id.erudit.org/iderudit/1007305ar

DOI : https://doi.org/10.7202/1007305ar

Aller au sommaire du numéro

Éditeur(s)

Les Éditions Historia Ecclesiæ Catholicæ Canadensis Inc.

ISSN

0318-6172 (imprimé)

1927-7067 (numérique)

Découvrir la revue

Citer cet article

Jaenen, C. J. (1968). Francisation et évangelisation des Amérindiens de la Nouvelle-France au XVIIe siècle. Sessions d'étude - Société canadienne d'histoire de l'Église catholique, 35, 33-46. https://doi.org/10.7202/1007305ar

Tous droits réservés @ Les Éditions Historia Ecclesiæ Catholicæ Canadensis Inc., 1968
Ce document est protégé par la loi sur le droit d'auteur. L'utilisation des services d'Érudit (y compris la reproduction) est assujettie à sa politique d'utilisation que vous pouvez consulter en ligne.

https://apropos.erudit.org/fr/usagers/politique-dutilisation/ 


\section{Francisation et évangélisation des Amérindiens de la Nouvelle-France au XVIIe siècle}

L'histoire des idées est relativement jeune. Plusieurs historiens en étudiant le commerce et les missions du Canada ont fait entrer les indigènes dans ces cadres d'interprétation historique. Je me propose de vous présenter les indigènes sous une nouvelle optique et de vous rappeler l'état actuel des travaux divers sur ce sujet.

En 1508, quelques sauvages, aujourd'hui appelés Amérindiens, furent amenés à Rouen où ils suscitèrent force curiosité. Quelques années plus tard, dans une lettre à François Ier, Jacques Cartier aborda la question de la possibilité de convertir "des gens sauvaiges sans connaissance de Dieu et sans usaiges de raison ${ }^{1}$ ". A cet appel à communiquer la « bonne raison et la bonne religion ", il n'y eut point de réaction immédiate en France si ce n'est des spéculations d'esprit. Les avis étaient fort partagés sur les Amérindiens. Selon l'école de Juan Ginés de Sepúlveda, juriste espagnol, les indigènes méritaient d'être cruellement subjugués et asservis (comme ils le furent en Amérique latine) à cause de leur triple bassesse: l'idolatrie, la barbarie et le paganisme. Il y avait, d'autre part, le point de vue de "l'homme naturel ", précurseur du noble sauvage de Rousseau, c'est-à-dire de l'aborigène inculte, incivilisé, fruste et grossier, mais capable d'être civilisé, soit un descendant d'Adam qui était un sujet digne d'être converti et assimilé à la vie française ${ }^{2}$.

Ce fut Henri IV qui demanda des missionnaires pour convertir les Amérindiens. Les Jésuites, qui avaient déjà sollicité la permission d'accompagner les flottes de pêche à la morue, relevèrent le défi ${ }^{3}$. Champlain

1 Archives Municipales de Saint-Malo, Série H.H., Carton I, 1; 3. L'arrivée des Amérindiens à Rouen est décrite dans Henry Harrisse, Découverte et Evolution cartographique de Terre-Neuve et des Pays circonvoisins (Londres, 1900), pp. 162-3, citant Henri Estienne, éd. Eusebii Caesariensis Episcopi Chronicon (Paris, 1512), f. 111 .

2 M. Menendez y Pelayo \& M. Garcia-Pelavy, éds., J. G. de Sepulveda : Tratado sobre las justas causas de la guerra contra los Indios (Mexico, 1941), pp. 101-5, 15l. Pour le point de vue opposé voir André LABrouQuÈre, La Notion $d^{\prime}$ assimilation en législation et économie coloniales (Hanoï, 1934), pp. 9, 28.

3 Lucien Campeau, s.j., La Première Mission d'Acadie (1602-1616) (Québec, 1967), docs. 2, 3, 5, pp. 4-8. L'aumônier de Marie de Medici a fait promettre 
écrivit en 1608 que les sauvages pourraient facilement se civiliser et se franciser et que, avec la langue française, ils acquerraient aussi un cœur et un esprit français. Il voyait les Amérindiens a vivans comme bestes brutes, \& croy que bien tost ils seraient reduits bon Chrestiens si on habitait leur terre ${ }^{4}$. Pour lui, évangélisation était synonyme de francisation. Le Père Allouez, lui aussi, exprimait en 1672, le point de vue optimiste tout en distinguant entre francisation et évangélisation :

Lo nom de Saurage fait naître une idée si décavantogeuse de ceur qui le portent, que bien des gens en Europe ont jugé qu'il était impossible d'en faire de véritables chrétiens...non seulement il y a de vrais Chrétiens parmi ces peuples sauvages, mais même qu'il y en a plus grand nombre à proportion que dans notre Europe civilisée ${ }^{5}$.

Montaigne était allé jusqu'à écrire au sujet du "devoir des peuples coloniaux " qu'il leur fallait "doucement polir et défricher » les indigènes en collaborant avec eux comme " nos égaux en tant qu'hommes ${ }^{6}$ ".

Deux attitudes de la part des Français sont à remarquer. Premièrement, la plupart des récits de voyages du $\mathrm{XVII}^{\mathrm{e}}$ siècle exaltent la beauté et la bonté, la candeur et la complaisance, l'hospitalité et l'honnêteté des Amérindiens ${ }^{7}$. Deuxièmement, vivement conscients et fiers de leur héritage culturel, les Français acceptent avec un plaisir manifeste la tâche d'élever des hommes brutes et grossiers vivant en milieu incivilisé au rang de Chrétiens raffinés de manières et instruits plus qu'à leur état

aux premiers missionnaires d'ériger au Nouveau-Monde un oratoire en honneur de saint Laurent. Archives Départementales de la Seine-Maritime (Rouen), Série G, liasse 6652 , fol. 1. La plus forte opposition semble avoir été celle de Robert du Pontgravé (le fils de François qui était étroitement lié à des marchands protestants d'Amsterdam pour le commerce du Canada - voir Gemeente Archief Amsterdam, Notarial Archives, passim), "non français mais turc, non turc mais athée ", selon Lescarbot. Le jeune Pontgravé s'opposa à la conversion des Amérindiens en accusant Poutrincourt d'être a un abuseur et qu'il les feroit mourir pour avoir leurs castors ". Marc LesCarbot, La Conversion des Sauvages qui ont esté baptizés en la Nouvelle France, cette année 1610 (Paris, 1610), pp. 23-24.

p. 163.

4 C. H. Laverdière, éd., Euvres de Champlain (Québec, 1870), tome III,

5 R. G. ThwaItes, ed., The Jesuit Relations and Allied Documents (Cleveland, 1896-1901), Vol. XVIII, p. 84.

6 Labrouquère, op. cit., p. 9; René Gonnard, La Légende du Bon Sauvage (Paris, 1946), pp. 40-70; voir aussi Gilbert ChInard, L'Amérique et le rêve exotique dans la littérature française au XVII ${ }^{\circ}$ et $X V I I I^{\circ}$ siècles (Paris, 1913), pp. 91-113; Lewis Henke, Aristotle and the American Indians (New-York, 1959); H. N. FaIrchild, The Noble Savage (New-York, 1928).

7 Pierre D'Avity, Description générale de l'Amérique, troisième partie du Monde (Paris, 1637), pp. 30-32. Le caractère apparemment très docile des Amérindiens suscite l'espoir de les convertir facilement. Voir Thomas LeFEvRe, Discours sommaire de la Navigation et du Commerce (Rouen, 1650), pp. 205-8; A. P. C., Série J, Journal en abrégé des voyages de Mons. d'asseline de Ronual s (1662). 
naturel des arts et du savoir « pour les tirer d'une habitude barbare à une vie civile et commode ${ }^{8}$. Le Sieur de Cobes écrivait en 1608 :

Quant au reste, pour leur façon de vivre, ils sont fort brutaux, mais ils commencent à se civiliser, \& à prendre noz mœurs, \& noz deportemens, ils se laissent facilement instruire à la foy Chrétienne, sans se montrer trop opiniâtres à leur Paganisme, tellement que s'il descendoit ça bas des Prédicateurs je croy qu'en peu de temps tout le pais se rendroit à la foy Chrétienne sans se faire autrement forcer, \& mesme que par la le chemin seroit ouvert en tout le reste de l'Amérique pour y faire la conqueste des ames, qui est plus que toutes les terres qu'on sçauroit iamais conquester $\boldsymbol{9}$.

Ces premiers contacts des Français avec les Amérindiens différaient sous quatre aspects des contacts antérieurs des Espagnols : premièrement, au $\mathrm{XVII}^{\bullet}$ siècle, le nationalisme était maintenant une force agissante: deuxièmement, le zèle contre-réformiste se faisait sentir; troisièmement, le contact s'effectuait surtout maintenant avec des peuplades seminomades et non sédentaires; enfin, la poursuite de la traite des fourrures encourageait le nomadisme indigène.

Les Français qui vinrent en Amérique du Nord étaient animés par une conception de l'ordre basée, comme ils le croyaient, sur des principes religieux immuables et éternels. Ils trouvèrent non pas un continent inoccupé, mais plutôt un hémisphère habité, ce qui posait deux problèmes : l'un théorique et l'autre pratique. En théorie, il leur fallait comprendre cet homme naturel, le sauvage. Dans la pratique, il leur fallait mater et dominer cet homme, l'intégrer à leurs mœurs économiques, politiques et culturelles, à leur conception de l'ordre. Ainsi Pierre de Gua, Sieur de Monts, avait-il pour instructions " de faire convertir, amener et instruire les peuples qui habitent cette contrée, de présent gens barbares, athées, sans foy de religion au Christianisme, \& en la créance \& profession de nôtre foy \& religion », instructions qui furent reprises et amplifiées par le vice-régent, le duc de Montmorency : " tacher d'en amener les nations à quelque profession de la foy Chrétienne, civilization de mœurs, règlement de leur vie, pratique \& intelligence avec les Français pour l'usage de leur commerce ${ }^{10}$. " Champlain adressa une requête à Louis XIII pour lui

8 Archives de l'Hôtel-Dieu, Québec, Lettres I, Lamy à la Rév. Mère Marie de St. Bonaventure, 20 mars, 1648.

9 Coppie d'une Lettre envoyée de la Nouvelle-France, ou Canada, par le Sieur de Cobes, Gentilhomme Poictevin, à un sien Amy (Lyon, 1609), pp. 13-14. Le Sieur Bertrand écrivait à Mons. de la Tronchaie, 25 juin, 1610 au sujet du * grand Sagamos, qui s'était converti : IIl promet faire baptiser les autres : autrement qu'il leur fera la guerre. s Lettre missive touchant la Conversion et Baptesme du grand Sagamos de la Nouvelle France (Paris, 1610), pp. 3-4.

10 Marc Lescarbot, Histoire de la Nouvelle-France (Paris, 1617), livres iv, pp. 212, 218. 
demander de l'aide financière au bénéfice d'un "séminaire " où seraient éduqués de jeunes Indiens qui aideraient, espérait-on, à convertir leurs aînés. En 1624, le Père Joseph le Caron écrivait:

....il faut espérer qu'à mesure que la Colonie se peuplera, nous humaniserons les Barbares; ce qui est premièrement nécessaire, leur esprit s'ouvrira \& le bon sens dont ils ont le fond: on les policera par les loix \& les manières de vivre à la Françoise, afin de les rendre capables d'entendre raison sur des Mystères si élever. Car tout ce qui regarde la vie humaine et crvile, sont des îiystères pour nos Barbares dans ïétar present, \& il faudra plus de dépense \& plus de travaux pour les rendre hommes qu'il n'en a fallu pour faire Chrestiens des Peuples entiers ${ }^{11 . . .}$

L'archevêque de Rouen, à l'occasion du baptême d'un jeune Huron de 16 ans (8 décembre 1627) qui avait fait ses études à Notre-Damedes-Anges, près de Québec, fit grand état de la conversion des indigènes ${ }^{12}$. Manifestement, on supposait que les Amérindiens assimilés à la culture française vénéreraient forcément le Dieu fait à l'image des Français. L'assimilation et la conversion s'envisageaient comme deux œuvres synonymes, mais les Récollets se virent bientôt contraints par l'expérience d'écarter cette hypothèse.

Les Jésuites qui leur succédèrent ne profitèrent point de l'expérience des Récollets; au contraire, ils agirent comme leurs devanciers. Ce n'est qu'en 1639 qu'ils changèrent de méthodes. Plusieurs innovations nettement canadiennes ne tardèrent pas à s'implanter: tout d'abord, les Jésuites se mirent à s'occuper d'enseignement élémentaire; puis, ils accueillirent des filles dans leurs classes; enfin, ils collaborèrent avec le clergé séculier et secondèrent le labeur missionnaire des religieuses en brousse ${ }^{13}$.

L'enthousiasme religieux qui régnait en France nourrissait le zèle évangélique de ces ambassadeurs de la culture en Nouvelle-France. Des fanatiques extatiques de la religion de la Compagnie du Très-SaintSacrement, groupement militant semi-secret, défilèrent un jour par les rues d'une ville normande en criant que la foi s'était en allée de France et que, eux, " les fous de Jésus Christ ", iraient dans le Nouveau-Monde

11 Chrestien Le ChercQ, Etablissement de la Foy dans la Nouvelle-France (Paris, 1691), pp. 264-5.

12 Bibliothèque Municipale, Le Havre, Manuscrits, Vol. 282, fol. 70. Il s'intéressait aussi à faire valoir ses droits sur le territoire canadien. Le 13 janvier, 1640 Richelieu donna les revenus d'une cinquième partie des a droits et concessions de la Compagnie de la Nouvelle France appartenant à Ravilly pour \& les séminaires qui seront establis pour la nourriture, instruction et entretenement des enfants des sauvages du dict paiz souz la conduite des pères Capucins . Candide le Nant, Pages glorieuses de l'Epopée canadienne (Paris, 1927), p. 313.

13 J. B. Conacher, ed., The History of Canada or New France by Father François du Creux, S. J. (Toronto, 1951), Vol. I, pp. 198-199. 
rebâtir Son royaume ${ }^{14}$. La plupart des Messieurs et Dames de la Société de Notre-Dame de Montréal pour la conversion des sauvages qui fondèrent Montréal en 1642 étaient associés à la Compagnie ${ }^{15}$ comme l'étaient aussi les gouverneurs d'Ailleboust et de Mézy, $\mathbf{M}^{\mathrm{gr}}$ de Laval, le curé de Bernières, plusieurs des séculiers, des Jésuites et des religieuses. Le dévotisme conviait les Français à réaliser en Amérique la pure et idéale société chrétienne. L'eschatologisme catholique évoquait la vision d'un Israël indien, du royaume millénaire ou de l'église spirituelle des mystiques par opposition à l'église institutionnelle corrompue de la Papauté, du Troisième Âge du Saint-Esprit ${ }^{15 a}$. $\mathrm{M}^{\mathrm{gr}} \mathrm{de}$ Laval et Marie de l'Incarnation voyaient la possibilité de rétablir la pureté de l'église primitive. Certains assimilaient la découverte du Nouveau-Monde à la fin imminente du monde et au retour du Christ. Que ces idées fussent répandues, on le voit bien dans le fait qu'un trafiquant de pelleteries et soldat comme Lamothe Cadillac ait cru fermement que les Amérindiens descendaient des dix tribus perdues d'Israël et qu'il ait écrit assez longuement à ce sujet ${ }^{16}$.

Le zèle évangélique s'est heurté à trois forces en particulier. Deux se rattachaient au caractère essentiellement matérialiste du comptoir commercial de la Nouvelle-France: la première tenait au problème créé par les nombreux Français qui ne pratiquaient pas les vertus exaltées par les missionnaires, et la seconde, à ce que quelques rares mais influents Français en France, en Nouvelle-France et surtout dans les colonies hollandaises et anglaises pratiquaient une forme protestante déviée de christianisme ${ }^{17}$. La troisième force d'opposition était le fruit de conceptions religieuses nativistes. Le missionnaire français était personnellement une énigme, son mode de vie était un mystère et sa doctrine était incompréhensible aux yeux de l'Amérindien. Abstinence, célibat et pauvreté apostolique étaient des concepts qui n'avaient guère de sens pour les membres des tribus nomades. Champlain avait dit d'eux "ils croyent que tous les songes qu'ils font sont véritables" et des missionnaires expérimentés mentionnaient dans leurs écrits (en 1670) " la Divinité du Songe, qui est le principe de toutes leurs erreurs,

14 Bibliothèque Municipale de Rouen, Papiers Féron, MS m. 276. Voir aussi Maurice Souriau, Le Mysticisme en Normandie au XVII' Siècle (Paris, 1923); E. R. AdaIr, France and the Beginnings of New France », Canadian Historical Review, Vol. XXV, No. 3 (September 1944), pp. 246-278; Raoul Allier, La Cabale des Dévots, 1627-1666 (Paris, 1902), pp. 10-17; abbé Albert Tessier, "La Compagnie du Saint-Sacrement ", Les Cahiers des Dix, No. VII (1942), pp. 27-44.

15 Marie-Claire Daveluy, La Société de Notre-Dame de Montréal, 1639-1663 (Montréal, 1965), pp. 17-45.

15a Norman Conn, The Pursuit of the Millenium (London, 1957), pp. 99-107.

16 Milton QuaIfe, ed., The Western Country in the 17th Century. The Memoirs of Antoine Lamothe Cadillac and Pierre Liette (New York, 1962), pp. 53-58. Aussi, Daniel Brinton, The Myths of the New World (New York, 1868).

17 Voir, par exemple, Ezechiel Carré, Echantillon de la Doctrine que les Jésuites enseignent aus Sauvages du Nouveau Monde (Boston, 1690), pp. 11. 
\& comme l'âme de leur Religion ${ }^{18}$ ". Ce n'est que depuis dernièrement que la valeur psychanalytique de ces " rêves symptômatiques d'apparitions》 est comprise des Européens ${ }^{19}$.

Le frère Sagard fut un des premiers à s'inquiéter de ce qui sortirait du contact entre une culture catholique, commerciale et acquisitive et une culture amérindienne communale, semi-nomade et primitive. Ses écrits parlent d'hommes "brutaux, athées et charnels, qui empesshoient la conversion et amendement de ce panvre neuple "; les Hurons, qui, par contraste, sont décrits comme ayant « l'esprit et l'entendement assez bon, et ne sont point si grossiers et si lourdauts que nous nous imaginons en France ${ }^{20}$ ". Il était fort confiant que "les sauvages mesmes les vagabons s'apprivoiseroient peu à peu " jusqu'au jour où les trafiquants français, décrits par le Père Jamet comme a grands Jureurs du St nom de Dieu » et «vivant en un grand libertinage " apparurent sur la scène pour contredire de la parole et par leur vie les exhortations des Franciscains ${ }^{21}$. LeFevre déclinait les facteurs ordinaires que devaient surmonter les missionnaires, soit la diversité de langues, les rivalités entre tribus, le nomadisme et la religion nativiste ${ }^{22}$.

Les Français imposèrent la traite des fourrures aux Amérindiens au $\mathrm{XVII}^{\circ}$ siècle, mais, au $\mathrm{XVIII}^{\circ}$, les Indiens en imposèrent le maintien aux Français comme condition d'alliance dans les guerres coloniales. En imposant leur commerce, les Français sapèrent les vues communales, l'économie et l'autorité tribale des Algonquiniens et introduisirent les débauches du trafic de l'eau-de-vie ${ }^{23}$. Des effets dysfonctionnels tels

18 Laverdière, op. cit., tome iii, p. 163. Voir aussi François du Creux, Historice Canadensis (Paris, 1664), Vol. I, p. 84. Pour des renseignements sur les Hurons voir, ThwaItes, op. cit., Vol. XXXIII, pp. 188-190, et Vol. LIV, pp. 64-74 sur les Iroquois. Noter, Ces Barbares montrent qu'ils sont capables d'écouter la raison \& de penetrer ses lumieres dans toute leur pureté; car quelques-uns des plus éclairez advouent qu'ils estoient convancus de ce que je leur disois \& qu'ils revenoient de la vanité de leurs songes. S Vol. LIV, p. 92.

19 Anthony F. C. Wallace, "Dreams and the Wishes of the Soul : A Type of Psychoanalytic Theory among the Seventeenth Century Iroquois s, American Anthropologist, Vol. 60 (1958), pp. 234-248.

20 Gabriel Sagard-Theodat, Le Grand Voyage du Pays des Hurons (Paris, 1632), pp. 178, 185.

21 A.P.C., B.N., 500 de Colbert, Vol. 483, Frère Denis Jamet au Cardinal de Joyeuse, 15 juillet, 1615, fol. 582.

22 LeFevre, op. cit., pp. 204-208; voir Harold Hickerson, \& The Feast of the Dead among the Seventeenth Century Algonkians of the Upper Great Lakes ", American Anthropologist, Vol. LXII (February 1960), pp. 81-107.

23 A. G. BaIley, "The Indian Problem in Early Canada ", America Indigena, Vol. II, No. 3 (July 1942), pp. 35-39; Alfred G. BAILEY, The Conflict of European and Eastern Algonquin Cultures, 1504-1700 (St. John, 1937). Une interprétation moins pessimiste du contact entre les deux cultures est B. G. TRIGGER, "Settlement as an Aspect of Iroquian Adaptation at the Time of Contact . American Anthropologist, Vol. 65 (1963), pp. 92-93. Pour ses vues sur le rôle des missionaires dans l'économie de la colonie voir. The Jesuits and the Fur Trade ", Ethnohistory, Vol. XII, No. 1 (1965), pp. 30-53. 
que la perte de la vie et des biens, la déviation sexuelle et la fureur agressive déclanchée par l'alcool s'en suivirent, effets qui furent accentués par le manque de moyens adéquats de contrôle social. La venue des Européens s'accompagna de maladies nouvelles qui revêtaient des proportions épidémiques, de l'épuisement du gibier et de l'intensification des guerres tribales. Le scalpement et les tortures intenses, par exemple, tout en tirant peut-être leur origine du culte du crâne et des sacrifices au soleil et au dieu de la guerre respectivement, s'étendirent des Iroquois (lorsque leur Confédération fut soumise à des pressions du fait des rivalités entre commerçants hollandais et français) aux Algonquins ${ }^{24}$. Il semble exister une corrélation directe entre la présence des Européens et l'introduction des armes à feu et l'intensification de la soi-disante barbarie indienne. Faut-il s'étonner que les Amérindiens aient craint la francisation et la christianisation? En outre, aux yeux de l'Indien, la bonne magie ou médecine constituait une sauvegarde de l'ordre moral et était hautement prisée, tandis que la mauvaise magie ou sorcellerie était la source des malheurs qui fondaient sur la tribu et elle était fort à éviter. L'association du baptême et de la mort, par exemple, inscrivait les missionnaires dans la catégorie des fauteurs de mal et des sorciers.

Quand ils se rendirent compte que les Français qui venaient en contact avec les Amérindiens adoptaient souvent le mode de vie des indigènes et que la tendance à l'« américanisation ", ou adaptation au milieu, se traduisait par une barbarisation progressive (comme le montra plus tard la baisse du niveau culturel en Nouvelle-France), les missionnaires cherchèrent à mettre en œuvre des mesures contraires. Ils adoptèrent le régime ségrégationniste du missionnaire franciscain Geronimo de Mendieta, régime qui a connu son achèvement dans les reducciones des Jésuites espagnols au Paraguay. La réserve fondée à Sillery en $1637^{25}$ a été la première version canadienne du dispositif ségrégationniste qui a subsisté jusqu'à nos jours sous une certaine forme.

24 П y a une littérature toujours accroissante sur ce sujet. Particulièrement utiles sont: William N. FENToN, a Problems arising from the Historic Northeastern Position of the Iroquois», Smithsonian Miscellaneous Collections, Vol. 100, pp. 159-251; Regina Flannery, An Analysis of Coastal Algongian Culture (Washington, 1939), p. 126; Martin Gusinde, Culte du crâne, têtestrophées et scalps », Revue Ciba, No. 63 (décembre 1963), pp. 2258-2283; A. Irving Hallowell, Some Psychological Characteristics of the Northeastern Indians ,, Man in Northeastern America (Cambridge, 1947), Vol. III, pp. 195-225; Diamond Jenness, The Indians of Canada (Ottawa, 1932), p. 279; Nathaniel KNowles, The Torture of Captives by the Indians of Eastern North America :, Proceedings of the American Philosophical Society, Vol. 82, No. 2 (March 1940), pp. 151-225. Pour des rapports de témoins voir Arthur T. Adams, ed., The Explorations of Pierre Esprit Radisson (Minneapolis, 1961), pp. 19-21 et J. TaIlhan éd., Mémoire sur les mours, coustumes et relligion des Sauuages de l'Amérique septentrionale par Nicholas Perrot (Leizig, 1864), pp. 146-7. 18617

25 Bibliothèque Nationale, Paris, MSS Français, $\mathrm{N}^{\mathrm{os}}$ 15619, 15620, 18112, 
Le système de réserves devait rendre les nomades sédentaires, les séparer et les isoler des influences perverses, et éduquer les "vrais croyants" pour en faire le noyau d'une cellule du nouvel Israël. Mesme le gouverneur Denonville avait quelque louange à formuler à l'endroit du système en 1685:

Je trouve tout ce que nous avons de sauvages establis dans les bourgades comme à Sillery, Lorette, au Sault de la prairie, a la Montagne de Montreal, tout cela Monseigneur est en vérité tenu dans une discipline

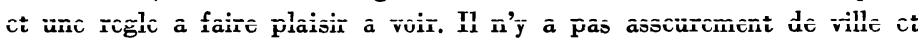
de vilage en France si bien reglés, qu'en tous ces lieux la; tant qu'il n'y a pas d'ivrognes qui arivent de nos habitation 26 .

Le missionnaire Bigot, au contraire, se plaignait que les Ind̋iens des réserves dits "domicilés " fussent la proie de l'ivrognerie et aussi de l'immoralité. Ce dont il ne se rendait pas compte, c'était que les Iroquois accueillaient l'eau-de-vie avec empressement, non pas tant pour son goût, mais pour s'intoxiquer et stimuler ainsi l'apparition des visions. Plus tard, l'alcool servit d'exutoire aux tensions, mais le concept onirique ne disparut jamais complètement ${ }^{2 \tau}$. Les docteurs de l'Université de Toulouse, dans une déclaration signée sur le trafic de l'eau-de-vie qui ne semble pas avoir été suffisamment documentée, portèrent un coup dur aux partisans de la ségrégation en observant que, même si certains indigènes domiciliés pouvaient mener une existence bien réglée, il était manifeste que les conversions n'étaient pas plus fréquentes chez eux que parmi les nomades ${ }^{28}$. Marie de l'Incarnation notait que la dislocation de la vie communale et de l'économie cynégétique primitive des Algonquins par les mœurs individualistes des trafiquants français était particulièrement grave ${ }^{29}$. Leurs institutions et leurs habitudes de vie traditionnelles furent perturbées avant que les missionnaires puissent remplacer la culture ancienne par les valeurs et les attributs plus substantiels de la culture européenne. Cela est demeuré essentiellement le "problème indien " du Canada. On pourrait ajouter que le fait que les réserves en vinrent à être peuplées de réfugiés indiens indique que la mission représentait un succédané à l'organisation indigène. Le succès avec lequel les Jésuites réussirent à imposer le catholicisme dans les réserves n'équivalait donc pas

26 A.P.C., Série C11A, Denonville au ministre, 13 novembre, 1685, Vol. VII, pp. 46-47. La thèse de Mendieta est élaborée dans J. L. Phelan, The Millenial Kingdom of the Franciscans in the New World (Berkeley, 1956), pp. 1-159.

27 Edmund S. CaRpenter, "Alcohol in the Iroquois Dream Quest *, American Journal of Psychiatry, Vol. CXVI, No. 2 (August 1959), pp. 148-151.

28 E. Reveillaud. éd., Histoire chronologique de la Nouvelle-France (Paris, 1888), p. 189.

29 Joyce Marshall ed., Word from New France. The Selected Letters of Marie de l'Incarnation (Toronto, 1957), pp. 76, 361. L'état déplorable où se trouvaient les Algonquins est décrit dans $A$. $A$. Q., Eglise du Canada, Rapport d'un missionnaire, 1671, Vol. VI, p. 9. 
exactement à une acculturation; ils ont plutôt ajouté le catholicisme à la couverture culturelle.

Plusieurs aspects de l'acculturation réalisée au $\mathrm{XVII}^{\circ}$ siècle sont mal compris. Les coureurs de bois, nouveau type social, ne furent pas longtemps les « interprètes " adolescents tels que Vignau. Marsolet et Brûlé que Champlain envoya vivre parmi les Amérindiens afin d'obtenir leur commerce et leur amitié. Ils furent (surtout après 1647) membres de familles qui cherchaient à arrondir le revenu familial. En fait, la traite des fourrures, en grande partie illicite, constituait le réel moyen de subsistance des familles immigrées de France et le grand moyen d'élévation sociale des Canadiens. Si on lit attentivement les Relations des Jésuites, on voit que les missionnaires français avaient tendance à adopter la langue, les coutumes, les manières, de leurs hôtes aborigènes et partageaient, dans une certaine mesure, leur vision (optique des choses). Quelques-uns devinrent, pour ainsi dire, des chefs religieux parmi les Amérindiens.

La ségrégation impliquait-elle nécessairement une discrimination préjudiciable? Les Français différaient-ils des Anglais ou des Hollandais par leur attitude vis-à-vis des Amérindiens ? Le cardinal Richelieu n'avait-il pas proclamé une certaine égalité en 1627 dans la charte de la Compagnie de la Nouvelle-France :

...que les descendans des François qui s'habitueront audit Pais ensemble les Sauvages qui seront amenés à la connoissance de la Foi et en ferront profession, seront censés \& reputés naturels François \& comme tels pourront venir habiter en France yuand bon leur semblera 30 ...

Il semble néanmoins que les Français aient pratiqué de la discrimination à l'endroit des Amérindiens en matière de préséance, de droits juridiques, de droits fonciers, de commerce, de fourniture d'armes à feu et de chevaux, et même de consultation à titre d'alliés ${ }^{31}$. Quand on examine

30 Etablissement de la Compagnie du Canada sous le titre de NouvelleFrance par les Articles des vingt-neuf Avril et sept May mil six cens vingt-sept (Paris, 1725), article xvii, p. 10; voir aussi Edict du Roy pour l'Establissement de la Compagnie de la Nouvelle-France (Paris, 1657); A.S.Q., Polygraphie III, $\mathrm{N}^{\circ} 2$; Archives Départementales de la Seine-Maritime, Série G, Liasse 2187, Plumatif du chapitre (Rouen), 8 décembre, 1627. Pour le point de vue du Cardinal Richelieu voir André Chevinlaud, Les Desseins de S. Em. de Richelieu pour l'Amérique, ce qui s'y passé de plus remarquable depuis l'établissement des colonies, et un ample traité du naturel, religion et moeurs des Indiens insulaires et de la terre ferme (Rennes, 1659). Voir de plus les études suivantes: William T. Hagan, The Indian in North American History (New York, 1963): Roy H. PEARCE, The Savages of America. A Study of the Indian and of the Idea of Civilization (Baltimore, 1953); Gilbert RoE, The Indian and the Horse (Norman, 1951) ; Frank C. HIBben, L'Homme primitif américain des origines préhistoriques à l'arrivée de l'homme blanc (Paris, 1953); Ruth Underhill, Red Man's America (New York, 1953).

31 Voir ma communication, . Problems of Assimilation in New France, 1603-1645 ", French Historical Studies, Vol. IV, No. 3 (1966), pp. 265-289. 
les quelques données fragmentaires qui existent sur les vues des Amérindiens sur les Français, il semble que le Français était un commerçant qui leur apportait armes à feu, munitions, ustensiles, couteaux, eau-de-vie et babioles [dont ils en étaient venus à dépendre] alors que l'Anglais du sud était un agriculteur qui dépossédait les tribus sédentaires. Ces deux types n'indiquent pas de différence fondamentale dans l'attitude des Anglais et des Français à l'égard des Amérindiens.

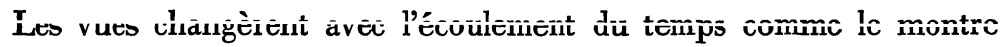
l'abandon du métissage. Champlain avait dit aux Hurons que, s'ils acceptaient la religion catholique apportée par les Récollets, les Français viendraient vivre parmi eux dans leurs villages, se marieraient à leurs filles et leur enseigneraient leurs arts et leurs métiers. C'était là une décision fort naturelle à prendre dans un établissement transmarin où prédominaient les hommes; elle ne tenait aucun compte de considérations élémentaires telles que l'influence de la mère dans la culture des enfants. Les pieux fondateurs de Montréal comptèrent eux aussi, en 1642, que le mariage entre races amènerait des conversions en masse, une augmentation marquée de la population coloniale et une mise en valeur rapide du potentiel agricole des terres basses du Saint-Laurent ${ }^{32}$. Les Amérindiens et les Français hésitaient, cependant, à régulariser leurs alliances. A maintes occasions, les Jésuites s'opposèrent ouvertement à de telles unions et les autorités civiles ne cessèrent de recommander le métissage, mais s'abstinrent d'y assurer de réelles incitations. Louis XIV donna instruction à l'intendant Bouteroue en $1668 \mathrm{~d}$ ' « employer toute l'autorité temporelle pour attirer les dits sauvages parmi les français, ce qui se peut faire par les mariages et par l'éducation de leurs enfants ", parce que les missionnaires avaient pour principe de tenir "les sauvages convertis dans leur forme de vie ordinaire ${ }^{33}$ ". Le roi, cependant, ne se servit pas d'incitations sous la forme, par exemple, de dots ou d'allocations ni n'adopta de politique générale d'assimilation.

Le rôle de l'État, on peut le voir dans l'intendance de Talon. A son retour à Québec à l'automne 1670, Talon fit mention de l'intérêt qu'il y avait pour le clergé à chercher \& des nouveaux sujets pour les élever dans nos mœurs, nos coutumes, notre langue et notre enseigne-

32 Les Véritables Motifs de Messieurs et Dames de la Société de NotreDame de Montréal (s.p. 1643), p. 107; François GIRY, La Vie de M. Jean Jacques Olier, prêtre, fondateur et premier Supérieur du Séminaire de Saint-Sulpice (Paris, 1687), pp. 98-99.

33 A.P.C., Série B, Instructions à Bouteroue, avril 1668, Vol. I, p. 83. Pour l'encouragement au métissage voir ThwaITes, op. cit., Vol. IX, pp. 216-218; Vol. X, p. 26; Vol. XIV, pp. 19-21; 263; aussi C. H. Laverdière \& H. R. CasGrain, éds., Le Journal des Jésuites (Québec, 1871), pp. 281, 312; Archivum Romanum Societatis Iesu, Rome, Gallia 109, fol. 3, Pierre de Sesmaisons : Raisons qui peuvent Induire Sa Sainteté a permettre aux Français... d'épouser des filles Sauvages.... 
ment » et suggéra à Colbert qu'il serait bon d'appuyer ce penchant " par deux ou trois lignes exprimant votre approbation". Se conformant presque avec soumission au vou de Talon, le Ministre de la Marine répondit le printemps suivant en faisant tenir des instructions que Talon transmit au clergé de la colonie:

Travailler tousjours par toute sorte de moyens à exciter tous les Ecclesiastiques et Religieux qui sont audit pays d'eslever parmy eux le plus grand nombre des dits enfants qu'il leur sera possible, affin qu'estant instruits dans les maximes de notre religion et dans nos mœurs ils puissent composer avec les habitans de Canada un mesme peuple, et fortiffier par ce moyen cette colonie là 34 .

$\mathrm{La}$ francisation, on la voyait comme un moyen de réaliser le peuplement souhaité par les esprits mercantiles tout en évitant, comme le craignait Louis XIV, de dépeupler la France par l'émigration ${ }^{\mathbf{3 5}}$.

Deux autres possibilités en dehors des idéaux de l'évangélisation et de la francisation s'étaient présentés dans les premières années du $\mathrm{XVII}^{\bullet}$ siècle, soit, d'une part, la destruction des Amérindiens comme Montaigne en avait donné l'avertissement ${ }^{36}$ et, d'autre part, la barbarisation des Français et leur assimilation à la société amérindienne. Le gouverneur Denonville signalait ce qui suit au ministre en 1685:

L'on a creu bien longtemps que l'approche des sauvages de nos habitations estoit un bien très considérable pour accoutumer ces peuples a vivre comme nous et a sinstruire de nre. relligion, je m'apercoy Monseigneur q. tout le contraire en est arivé car au lieu de les acoutumer a nos Loys, je vous asseure qu'ils nous communiquent fort tout ce qu'ils ont de plus mechant, et ne prennent eux mesmes q'ce qu'il y a de mauvais et de vitieux en Nous ${ }^{37}$.

Comme le commerce français et les missions catholiques sapaient les facteurs de stabilisation que renfermait la culture indigène, l'idée que les Amérindiens étaient des barbares avides de sang, malpropres et dépravés en vint à remplacer la notion du " bon sauvage ». A son arrivée au Canada, $\mathrm{M}^{\mathrm{gr}}$ de Laval manifesta un intérêt peu commun pour les chrétiens indigènes, montra de la préférence pour leur parti-

34 A.P.C., Série B, Instructions à Talon, février 1671, Vol. III, pp. 66-67; P. Margry, éd., Découvertes et Etablissements des Français dans l'ouest et dans le sud de l'Amérique septentrionale (Paris, 1879), Talon à Colbert, 10 novembre, 1670, Vol. I, p. 92.

35 Voir ma communication \& Le Colbertisme ", Revue d'histoire de l'Amérique française, Vol. XVIII, $\mathrm{N}^{\circ} 1$ (1964), pp. 64-84, Vol. XVIII, $\mathrm{N}^{\circ} 2$ (1964), pp. $252-266$.

36 Donald H. Frame, ed., The Complete Works of Montaigne. Essays, Travel Journal, Letters (Stanford, 1957), livre iii, p. 693. Le même point de vue se trouve dans Chrestien LE CLercQ, Nouvelle Relation de la Gaspésie (Paris, 1691), pp. $267 \mathrm{ff}$. pp. $46-47$.

37 A.P.C., Série C11A, Denonville au ministre, 13 novembre, 1685, Vol. VII, 
cipation aux cérémonies religieuses (à la grande consternation des colons blancs) et alla jusqu'à faire sonner les cloches de l'église à la mort d'une convertie indienne, ce "quı ne se fait jamais à la mort d'un sauvage " remarquait le supérieur des Jésuites ${ }^{38}$. A la vie sédentaire, aux réserves établies à part et au métissage s'ajouta un autre moyen, l'éducation intégrée. Dans la pratique, cependant, les religieux et religieuses isolèrent Européens et Amérindiens dans la plupart des érnles ${ }^{39}$. Pêl d'années anrès son arrivée. $M^{\mathrm{gr}}$ de Laval bénissait une croisade lancée contre les Iroquois, ces "turcs" qui ne méritaient que l'anéantissement, et finit par exclure les jeunes Indiens de son école de métiers de Saint-Joachim. Vière Marie de l'Incarnation appuyait ces vues:

On ne les met pas pourtant en la bourgade des Français, crainte qu'ils n'en imitent, mais les sauvages ne sont pas capables de la liberté française quoique honnête ${ }^{40}$.

C'est une pratique qui suivait tout au plus la maxime "séparés mais égaux ».

La politique de la francisation échoua dans une certaine mesure parce qu'elle n'était pas pratique. Marie de l'Incarnation commentait comme il suit l'insistance que mettait Louis XIV à demander un redoublement d'efforts en vue d'assimiler les indigènes :

Si Sa Majesté le veut, nous sommes prêtes de le faire par l'obéissance que nous lui devons, et surtout parce que nous sommes toutes disposées à faire ce qui sera à la plus grande gloire de Dieu. C'est pourtant une chose très difficile, pour ne pas dire impossible, de les franciser ou civiliser. Nous en avons l'expérience plus que tout autre, et nous avons remarqué que de cent de celles qui ont passé par nos mains à peine en avons nous civilisé une. Nous y trouvons de la docilité et de l'esprit, mais lors qu'on y pense le moins, elles montent par dessus notre clôture et s'en vont courir dans les bois avec leurs parents, où elles

38 Journal des Jésuites, p. 224; Thwaites, op. cit., Vol. LII, p. 244. Voir pour plus de détails Journal des Jésuites, pp. 17-18, 20, 31, 102-103, 122, 126, 139-140; A.S.Q., Lettres $N, \mathrm{~N}^{\circ} 5$; Sacra Rituum Congregatio, Sectio Historia, Quebecen. Beatificationis et Canonizationis Ven. Servi Dei Francisci de Montmorency Laval (Rome, 1961), Docs. xviii, xxiii, pp. 37-39. Pour la question de changement de point de vue voir C. Martin, Lettres de la Vén. Mère Marie de l'Incarnation (Paris, 1681), pp. 387, 557-8. pp. 141-142.

39 Au sujet des Capucins en Acadie voir Candide lE Nant, op. cit.,

40 Au Roi. Raisons qui font voir combien il est important au roi et $\grave{a}$ son Etat de défendre ses sujets de la Nouvelle-France, dit Canadas, contre les invasions des Iroquois (s.p., s.d.) nous montre comment une opinion publique, fut créée au Canada pendant les 1650. Dom Albert JAMET, éd., Marie de l'Incarnation, Ursuline de Tours, Ecrits Spirituels et Historiques (Paris, 1935). Vol. III, p. 378. 
trouvent plus de plaisir que dans tous les agréments de nos maisons françaises $\mathbf{4 1}$.

Les Ursulines n'avaient encore réussi à éduquer que 7 ou 8 filles en 1685 , filles qui se marièrent à des Français et adoptèrent la vie sédentaire dans les établissements. Ni l'Église ni l'État ne fournissaient assez d'hommes, d'argent ou de matériaux pour vêtir et loger tous les Indiens à la façon européenne, les entourer d'exemples de la vie civile française et assurer des moyens d'instruction au bénéfice de tous les jeunes indigènes. Ces mesures, elles étaient impensables au $\mathrm{XVII}^{\mathrm{e}}$ siècle, mais elles n'en étaient pas moins les conditions nécessaires à l'assimilation de toute une population aborigène.

La politique de francisation échoua dans une certaine mesure parce que les Amérindiens entendaient survivre, possédaient un sens profond de leur identité et constituaient un peuple sûr de lui, indépendant, autonome et bien adapté dans le milieu canadien. Sagard avait dit au début du siècle que les Amérindiens estimaient leurs enfants plus sages et plus intelligents que les Français qu'ils considéraient très bornés par comparaison avec eux-mêmes ${ }^{42}$. De l'avis de Peter Kalm, les Indiens étaient trop fiers pour apprendre le français! Quoi qu'il en soit, qu'avaient-ils besoin de le faire puisque trafiquants et missionnaires apprenaient leur propre langue?

La christianisation a-t-elle mieux réussi que la francisation ? On peut noter que quelques Amérindiens furent du nombre des martyrs, mais que les saints furent sans exception des Français. Il n'y eut même pas de Canadiens parmi eux. Au dire du Père Lalement, les conversions se chiffrèrent par environ 10,600 au cours d'une quarantaine d'années, soit un nombre qui semble peut-être assez impressionnant, mais qui ne représentait qu'une faible proportion de l'ensemble de la population des tribus évangélisées ${ }^{43}$. Deux milliers environ de ces conversions furent des baptêmes faits à l'article de la mort. Il y eut des mystiques indiens et certains des néophytes, certes, pratiquaient la flagellation et « mêlaient leur sang à leurs larmes » durant les célébrations de la Semaine Sainte ${ }^{44}$. Mais des prêtres, des religieuses ou des enseignants amérindiens, il n'y en eut point.

41 L'abbé Richaudeau, Lettres de la Révérende Mère Marie de l'Incarnation (Tournai, 1876), Vol. II, p. 372. Saint-Vallier s'exprima clairement sur le même sujet - A.S.Q., Lettres $P$, No 47 , Saint-Vallier à Louis XIV, 1686.

42 George M. Wrong, ed., The Long Journey to the Country of the Hurons (Toronto, 1939), pp. 139-140.

43 L. Pouliot, Etude sur les Relations des Jésuites de la Nouvelle-France (Montréal, 1940), p. 233 indique qu'il y eut 16,014 conversions entre 1632 et 1672 , les années les plus fructueuses 1649 et 1650 .

44 Léon Pouliot, Etats mystiques chez les Convertis Indiens dans la Nouvelle-France", Société canadienne d'histoire de l'Eglise catholique, Rapport 1939-1940 (1940), pp. 99-106. Une Huronne fut admise à prendre l'habit de 
La conclusion qui s'impose, c'est que les Français, groupe minoritaire dispersé sur un vaste territoire et moins bien adapté au milieu canadien à bien des égards que les indigènes, étaient trop souvent euxmêmes assimilés par la société amérindienne. Voici ce qu'en disait Nicolas Perrot :

tellement que ces Canadiens se rendirent semblables aux sauvages dont ils copièrent si bien le libertinage qu'ils oublièrent ce qu'ils (devoient) à la subordination et descipline françoise, et, si je l'ose dire, au Cinristianisme mesme ${ }^{4} \mathrm{x}$.

La présence des colons et trafiquants européens empêchait la christianisation ou l'évangélisation. L'acculturation était donc souvent un processus d'américanisation, non pas de francisation.

\section{Cornelius J. JaEnen, Ph.D.. Professeur agrégé, Université d'Ottawa.}

l'Hôtel-Dieu de Québec en 1657; après huit mois de postulat, elle tomba malade et on lui fit faire ses vœux; elle mourut deux jours plus tard. L'annaliste de l'HôtelDieu écrivit : * C'est la première et la seule Sauvagesse jusqu'à présent [1716] à qui Dieu ait accordé la grâce de la vocation religieuse." Le problème ne semble jamais avoir été résolu. Voir Frederick Eberschweiler, "An Indian Clergy Impossible , Catholic World, Vol. 65 (1897), pp. 815-824; L. W. ReILly, « Why there is no Indian Priest ", American Ecclesiastical Review, Vol. IV (1890), pp. 267-280

45 J. Tailhan, éd., Mémoire sur les Mours, coustumes et relligion des Sauuages de l'Amérique septentrionale par Nicholas Perrot (Leipizig, 1864), pp. 130-131. Voir les œuvres suivantes pour l'influence du milieu nord-américain sur les Européens; E. Russell Carter, The Gift is Rich (New York, 1955); R. F. HeIzer, *The American Indian : background and contributions ", dans F. J. Brown \& J. S. Roucek, éds., One America (New York, 1952); A. F. ChalMBERLIN, "The Contributions of the American Indian to civilization ", Proceedings of the American Antiguarian Society, Vol. 16 (1905), pp. 91-126; Felix S. CoHEN, - Americanizing the White Man », The American Scholar (Spring 1952); E. E. EDWARDS, *American Indians' contribution to civilization ", Minnesota History, Vol. XV (1934), pp. 255-272; Leo J. Frachtenberg, "Our Indebtness to the American Indian», Wisconsin Archaeologist, Vol. XIV (1914), pp. 64-69; A. Irving Hollowell, "The Impact of the American Indian on American Culture *, American Anthropologist, Vol. 59 (1957), pp. 201-217; Diamond Jenness, - Canada's Debt to the Indians ", Canadian Geographical Journal, Vol. 18 (1939), pp. 268-275; Clark WISsLER, "The Influence of aboriginal Indian culture on American life ", dans Alain Locke \& B. J. Stern, éds. When Peoples Meet $(\mathrm{New}$ York, 1946); Clark WIssler, "Contributions of the American Indian , dans F. J. Brown \& J. S. RouceK, éds., Our Racial and National Minorities (New York, 1937). 